\title{
The ORCHIDS Intrusion Detection Tool ${ }^{\star}$
}

\author{
Julien Olivain Jean Goubault-Larrecq \\ LSV/CNRS UMR 8643 \& INRIA Futurs projet SECSI \& ENS Cachan \\ 61 avenue du président-Wilson, F-94235 Cachan Cedex \\ olivainelsv.ens-cachan.fr \\ Phone: +33-14740 7550 Fax: +33-1 47402464
}

\begin{abstract}
ORCHIDS is an intrusion detection tool based on techniques for fast, on-line model-checking. Temporal formulae are taken from a temporal logic tailored to the description of intrusion signatures. They are checked against merged network and system event flows, which together form a linear Kripke structure.
\end{abstract}

Introduction: Misuse Detection as Model-Checking. ORCHIDS is a new intrusion detection tool, capable of analyzing and correlating events over time, in real time. Its purpose is to detect, report, and take countermeasures against intruders. The core of the engine is originally based on the language and algorithm in the second part of the paper by Muriel Roger and Jean Goubault-Larrecq [6]. Since then, the algorithm evolved: new features (committed choices, synchronization variables), as well as extra abstract interpretation-based optimizations, and the correction of a slight bug in op.cit., appear in the unpublished report [1]. Additional features (cuts, the "without" operator) were described in the unpublished deliverable [2]. Finally, contrarily to the prototype mentioned in [6], ORCHIDS scales up to real-world, complex intrusion detection.

The starting point of the ORCHIDS endeavor is that intrusion detection, and specifically misuse detection, whereby bad behavior (so-called attacks) is specified in some language and alerts are notified when bad behavior is detected, is essentially a modelchecking task. The Kripke model to be analyzed is an event flow (collected from various logs, and other system or network sources), and complex attack signatures are described in an application-specific temporal logic.

Let us give an example of a modern attack [5]. Far from being a gedankenexperiment, this really works in practice and has already been used to penetrate some systems. We also insist that, as systems get more and more secure, we are faced with more and more complex attacks, and [5] is just one representative.

The schema on the right displays what a modular kernel (e.g., Linux) does when a user program (here with pid 100) calls an unimplemented functionality.

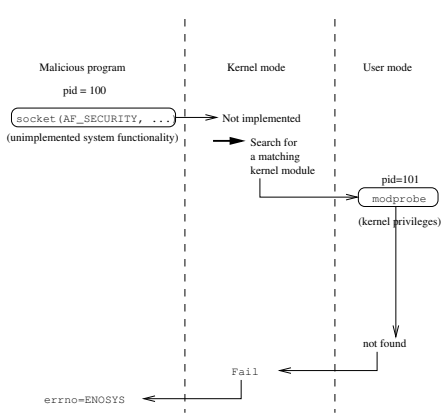

\footnotetext{
* Partially supported by the RNTL Project DICO, the ACI jeunes chercheurs "Sécurite informatique, protocoles crypto. et détection d'intrusions" and the ACI cryptologie "Psi-Robuste".
} 
The kernel will search for a kernel module that implements this functionality, calling the modprobe utility to search and install the desired module. If modprobe does not find any matching module, an error code is reported to the user program.

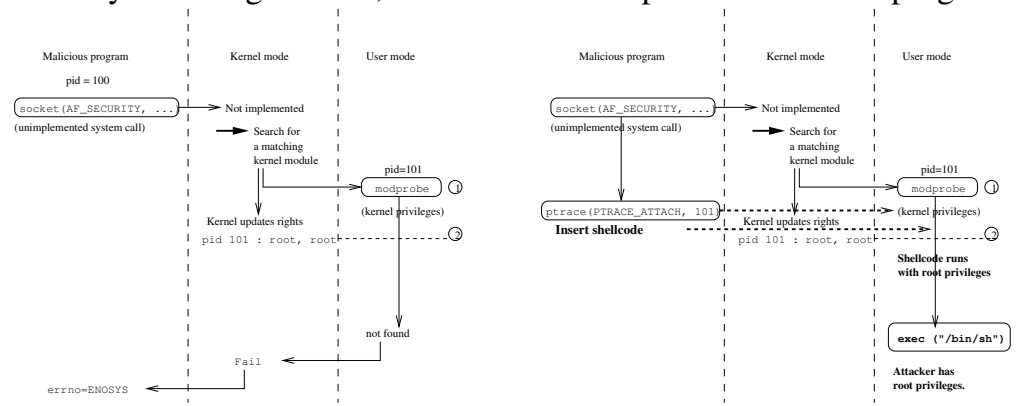

While this is how this is meant to work, some versions of Linux suffer from a race condition (above, left): while modprobe has all kernel privileges, the kernel updates the owner tables to make modprobe root-owned while modprobe has already started running. So there is a small amount of time where the malicious program has complete control over the kernel process modprobe: between timepoints (1) and (2). The malicious program takes this opportunity to attach the modprobe process through the standard Unix debugging API function ptrace, inserting a shellcode (malicious code) inside modprobe's code. When modprobe resumes execution, it will execute any code of the intruder's choosing, with full root privileges (above, right).

Challenges in On-Line, Real-Time Model-Checking. Intrusion detection requires specific logics to describe attack signatures, and specific model-checking algorithms.

Compared to standard misuse detection tools, a temporal logic allows one to describe behavior involving several events over time: standard misuse detection tools (e.g., anti-virus software or simple network intrusion detection systems) match a library of patterns against single events, and emit an alert once single so-called dangerous events occur. More and more attacks nowadays involving complex, correlated sequences of events, which are usually individually benign. In the ptrace attack, no individual event (calling an unimplemented system call, or ptrace, etc.) is dangerous per se.

The signature language of ORCHIDS extends [6, Section 4]. Among other things, it allows one to write temporal formulas of the typical form $F_{1} \wedge \diamond\left(F_{2} \wedge \diamond\left(F_{3} \ldots\right) \vee\right.$ $\left.F_{2}^{\prime} \wedge \diamond\left(F_{3}^{\prime} \ldots\right)\right)$, where $\diamond$ is the strict "there exists in the future" operator. In general, more complex formulae can be written, using operators resembling Wolper's ETL [7]— except going through a transition denotes either no time-passing at all ( $\epsilon$-transitions), or $\diamond$ (not $\bigcirc$ as in ETL). Such formulae are described internally as automata; we just give a signature for the ptrace exploit as an illustration. (Some other attacks such as the do_brk exploit [3] require committed choices, or other features of ORCHIDS not described in [6]. To save space, we don't recount them here.) A formula matching the ptrace exploit is the following automaton, described in slightly idealized form:

$$
\begin{aligned}
\operatorname{Attach}(X, Y, Z)-\diamond \rightarrow \operatorname{Exec}(Y)-\diamond & \operatorname{Syscall}(X, Y)-\diamond \rightarrow \operatorname{Getregs}(X, Y) \\
\diamond & \text { Poketext }(X, Y)-\diamond \rightarrow \operatorname{Detach}(X, Y)
\end{aligned}
$$

where $X, Y, Z$ are existentially quantified first-order variables meant to match the attacker's pid, the target's pid (i.e., modprobe's pid), and the attacker's effective uid 
respectively; where $\operatorname{Attach}(X, Y, Z)$ abbreviates a formula (not shown) matching any single event displaying a call to ptrace by process $X$ owned by $Z$, on process $Y$, with the ATTACH command, $\operatorname{Exec}(Y)$ matches single events where / sbin/modprobe is execed with pid $Y$, and the remaining formulas match single events where process $X$ issues a call to ptrace on target $Y$, with respective commands SYSCALL, GETREGS, POKETEXT (used to insert the shellcode), and DETACH.

Compared to other more standard uses of model-checking, the logic of ORCHIDS is constrained to only specify eventuality properties. This is because the model-checker needs to to work on-line, that is, by always working on some finite (and expanding over time) prefix of an infinite sequence of events. Compared to standard model-checking algorithms, e.g., based on Büchi automata for LTL, the model-checker is not allowed to make multiple passes over the sequence of events (e.g., we cannot rebuild a product automaton each time a new event is added); in general, intrusion detection tasks are submitted to very stringent efficiency requirements, both in time and in space.

Second, the logic of ORCHIDS includes some first-order features. As witnessed by the use of variables $X, Y, Z$ in (1), this logic can be seen as an existential fragment of a first-order temporal logic.

Finally, such a model-checker cannot just report the existence of matches, but must enumerate all matches among a given representative subset, with the corresponding values of the existential variables, build an alert for each match and possibly trigger countermeasures. This is the raison d'être behind the Getregs $(X, Y)$ formula in (1); if we only wanted a yes/no answer, this would just be redundant, and could be erased from the automaton; here, this is used to be able to report whether the attacker issued at least one call to ptrace(PTRACE_GETREGS) or not during the attack.

The model-checking task for the logic of ORCHIDS is NP-hard (it includes that of [6, Section 4]), but can be done using an efficient, on-line and real-time algorithm [2, 1]. Moreover, this algorithm is optimal in the following sense: for every attack signature (formula $F$ ), if at least one attack (sequence of possibly non-contiguous events) is started at event $e_{0}$ that matches $F$, then exactly one attack is reported amongst these, the one with the so-called shortest run. The latter is usually the most meaningful attack among all those that match. The notion of shortest run was refined in ORCHIDS, and now appears as a particular case of cuts [2]; this gives more control as to which unique attack we wish to isolate amongst those that match.

Related Work. There are many other formalisms attempting to detect complex intrusion detection scenarios, using means as diverse as Petri nets, parsing schemata, continuous data streams, etc. Perhaps one of the most relevant is run-time monitoring (or cousins: F. Schneider's security automata and variants, and security code weaving), where the event flow is synchronized at run-time with a monitor automaton describing paths to bad states. The ORCHIDS approach is certainly close to the latter (although arrows in e.g., (1) are more complex than simple automaton transitions); shortest runs and cuts, which introduce priorities between paths in the monitor, and the fact that only one optimal path among equivalent paths is reported, is a useful refinement.

Implementation. The ORCHIDS engine is implemented in C. At the core of ORCHIDS lies a fast virtual machine for a massively-forking virtual parallel machine, and a bytecode compiler from formulae (such as (1)) to this virtual machine. ORCHIDS uses a 
hierarchy of input modules to subscribe to, and to parse incoming events, classified by input source and/or event format. A main event dispatcher reads from polled and realtime I/O, reads sequences of events in syslog format, snare, sunbsm, apache and other various formats, coming from log files or directly through dedicated network connections, and feeds the relevant events to the core engine. ORCHIDS is able to do both system-level and network-based intrusion detection, simultaneously.

Here are a few figures of ORCHIDS on an instance of the ptrace attack:

\begin{tabular}{r|r}
\hline \hline Time : & Real time : $1267 \mathrm{~s}$ \\
& CPU Time : $370.810 \mathrm{~s}$ \\
& CPU usage : $29.27 \%$ \\
\hline \hline Resources : & Memory (peak) : $2.348 \mathrm{MB}$ \\
& Signalisation network load : $1.5 \mathrm{~GB}$ \\
\hline \hline Analyzer : & Loading and rule compilation : $<5 \mathrm{~ms}$ \\
& Processed events : 4058732 \\
\hline \hline
\end{tabular}

To stress the detection engine, the attack was hidden in the middle of a huge amount of normal ptrace debugging events, generated by tracing the compilation of the whole GCC C Compiler (with the command line tar xzvf gcc-3.3.2.tar.gz; cd gcc-3.3.2 ; . /configure; $\mathrm{cd}$ gCc ; strace -F -f make).

Conclusion. The ptrace attack above is one of the typical attacks that ORCHIDS can detect. Experiments are going on at LSV to test ORCHIDS on actual network traffic and system event flows.

From the point of view of security, a good news is that, contrarily to most misuse intrusion detection systems, ORCHIDS is able to detect intrusions that were not previously known (contrarily to popular belief on misuse IDSs). E.g., the signature we use for the do_brk attack [3], which tests whether some process managed to gain root privilege without calling any of the adequate system calls, detected the recent (Jan. 2005) Linux uselib attack.

For more information, see the Web page [4].

\section{References}

1. J. Goubault-Larrecq. Un algorithme pour l'analyse de logs. Research Report LSV-02-18, Lab. Specification and Verification, ENS de Cachan, Cachan, France, Nov. 2002. 33 pages.

2. J. Goubault-Larrecq, J.-P. Pouzol, S. Demri, L. Mé, and P. Carle. Langages de détection d'attaques par signatures. Sous-projet 3, livrable 1 du projet RNTL DICO. Version 1, June 2002. 30 pages.

3. A. Morton and P. Starzetz. Linux kernel do_brk function boundary condition vulnerability. http: //www.securityfocus.com/bid/9138, Dec. 2003. References CAN-20030961 (CVE), BugTraq Id 9138.

4. J. Olivain. ORCHIDS - real-time event analysis and temporal correlation for intrusion detection in information systems. http: //www. lsv.ens-cachan.fr/orchids/, 2004.

5. W. Purczyński. Linux kernel privileged process hijacking vulnerability. http://www . securityfocus. com/bid/7112, Mar. 2003. BugTraq Id 7112.

6. M. Roger and J. Goubault-Larrecq. Log auditing through model checking. In Proc. 14th IEEE Computer Security Foundations Workshop (CSFW'01), Cape Breton, Nova Scotia, Canada, June 2001, pages 220-236. IEEE Comp. Soc. Press, 2001.

7. P. Wolper. Temporal logic can be more expressive. Information and Control, 56(1/2):72-99, 1983. 


\section{A Appendix}

\section{A.1 Tool demonstration}

The demonstration will emphasize the most important aspects of the intrusion detection tool ORCHIDS. Our emphasis is to show that slightly deviant model-checking tasks can profitably be used for actual security applications, in real time, detecting real, sophisticated, and hard-to-detect attacks.

Our aim in demonstrating ORCHIDS is not as a proof-of-concept for some modelchecking algorithm. The stress is not on algorithmic novelty (although ORCHIDS implements new things such as committed choices, or cuts, or the "without" operator), rather on the usability of an actual tool based on some ideas in model-checking. This is in particular why we submit this as a tool presentation. Because this is a tool presentation, we have chosen to stress its application domain, and the challenges it has to meet, rather than any fancy algorithmic feature that had better be presented in a regular paper.

Of course, this does not mean that we won't describe any of the fancy new features of ORCHIDS if asked so, in particular compared to the proof-of-concept algorithm of [6]: committed choices, cuts, notably. We just feel that it is not the purpose of a tool presentation to stress these points.

In addition to the points stressed in the tool paper itself, and in particular the challenges that have to be met for an on-line, real-time model-checker in such specific environments, we want to show:

The large range of data sources and types: ORCHIDS can extract data and events from multiple distributed sources (kernel system calls, network firewall actions, web server, mail server, etc.) This wide variety of events enables ORCHIDS to correlate many events over time and over different event sources, a particularly welcome asset in a modern intrusion detection system. The demonstration shows the modularity of ORCHIDS; it currently recognizes 400 data fields from about 50 data sources.

On-line analysis: ORCHIDS continuously receives events from sources, as soon as possible after their creation date (the delay depends of the communication method for reporting events, either local or remotely, through udp). The analysis has to be done in one pass, incrementally, over the event flow. The event flow, as seen by the analyzer, will be shown during the demonstration.

Real-time detection and coutermeasure: ORCHIDS collects data, computes information, and executes actions at any given instant of the detection process. ORCHIDS is organized so as to react as quickly as possible to an attack, and must therefore announce that an attack has been detected as soon as the last characteristic event of the attack has been received. Additionally, ORCHIDS is able to generate intermediate reports, when only part of a temporal formula has been matched, i.e., when it is only likely that a given attack is currently being perpetrated. If the temporal formula eventually matches in whole, ORCHIDS will eventually confirm that the attack occurred, through a complementary report.

Moreover, we shall demonstrate that ORCHIDS is able to take countermeasures against attackers by killing compromised processes, closing suspect user accounts, or blocking network connections by inserting new rules into a firewall. 
These features will be demonstrated by running ORCHIDS itself, and by inspecting the formulae, the currently monitored instances of these formulae (the threads), the current event flow, through ORCHIDS' administration interface (see Figure 1).

It is to be noted, too, that the attacks we shall demonstrate are no toy examples. They are recent, sophisticated attacks, which have already been used in practice (by hackers), and whose detection is particularly challenging.

The demonstration is done on a single laptop. Demonstrating a distributed intrusion detection system working on just one computer entails some specific difficulties or deviations from real behavior:

- The analyzer runs on the audited computer; so it will analyze its own event flow, as well as its sensors'. Some precautions have to be taken to avoid the analyzer to loop, analyzing itself and therefore generating new events that have to be monitored,..., recursively.

- The real attacks we shall demonstrate are launched against the operating system kernel, which can then end up in an unstable state. We have been careful to craft our attacks so as to be perfect (from the attacker point of view), i.e., to steal the target resource without crashing the whole system. In actual deployments (and in our testbed at LSV), ORCHIDS would naturally be running on a remote machine with only in connections to receive event flows, so that crashing an audited system would not crash ORCHIDS.

- The activity of a single host is much less intense than that of an entire network. To simulate the event flow in a realistic network environment, we shall use the EvtGen tool (which we designed specifically to this end) to generate fake events, too, simulating normal behaviour with randomly interspersed errors or anomalies.

The figure on the right shows how differents components interact on the demonstration computer.

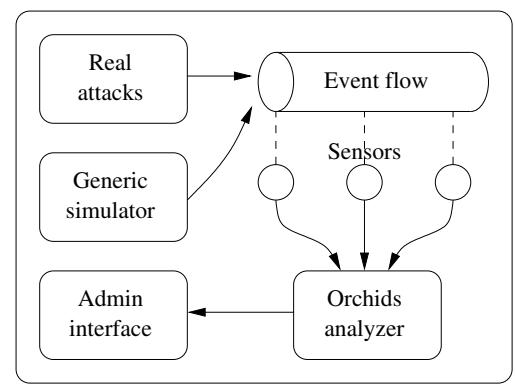

The complete presentation of the tool consists in launching a few attacks, once with ORCHIDS disabled to show that the attacks really work and allow us to gain illegitimate root access; and a second time to show that the same attacks will be detected and countered by ORCHIDS. We do not require any specific equipment other than a videoprojector; we shall use a laptop of our own, with all necessary tools installed.

We illustrate just one attack here, corresponding to the one described in the paper. Figure 2 shows an intruder logging in through ssh on a machine (which will just be localhost in the demonstration), compiling a ptrace attack and getting root privileges. (As the \# sign at the final prompt shows.) Note that the attack is particularly verbose, for pedagogical reasons, and explains every step it does: see the lines starting with $[+]$. (An actual attack would not be as verbose.) 


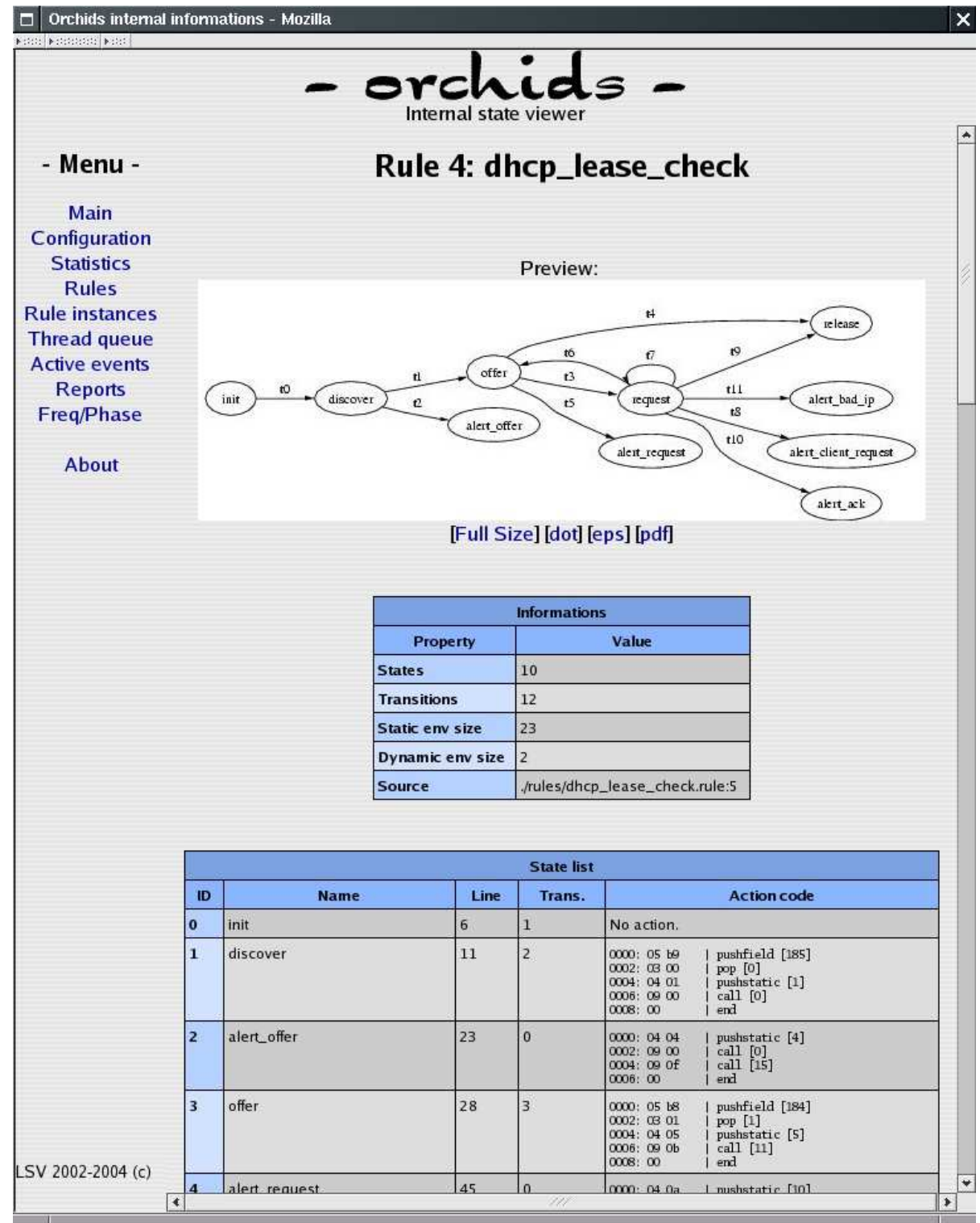

Fig. 1. The ORCHIDS administration interface 


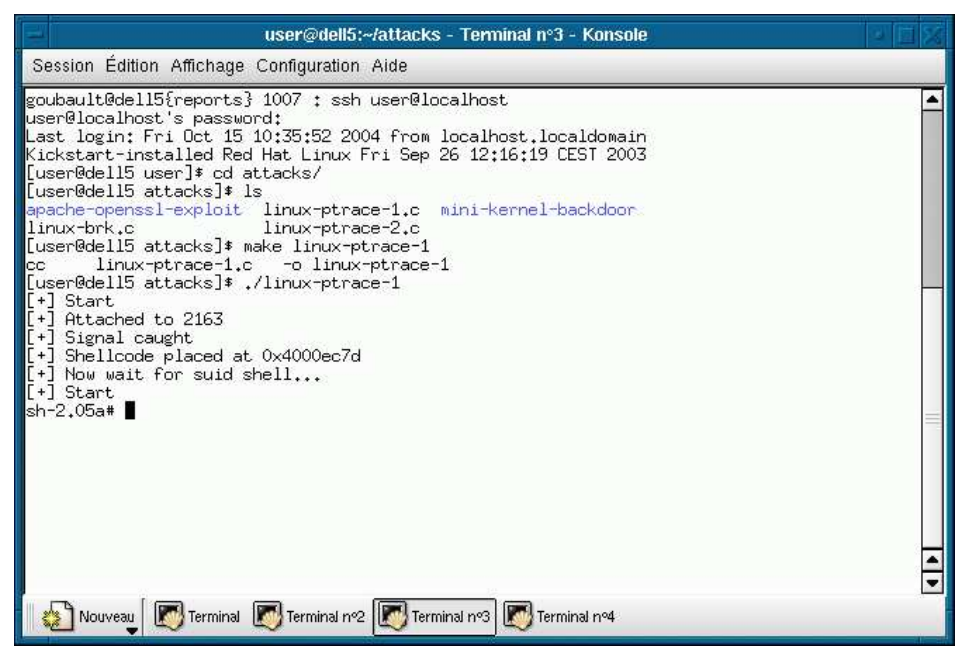

Fig. 2. Running a ptrace attack without ORCHIDS

goubault@dell5.Isv.ens-cachan.fr: Momelgoubaulttorchids-localdemo-2004-10 - Temminal n³ - Kol

Session Édition Affichage Configuration Aide

goubaultadell5\{reports\} 1009 : ssh user[localhost

user@localhost's password:
Last login: Fri Oct 15 10:36:49 2004 from localhost. localdomain

ickstart-installed Red Hat Linux Fri Sep 26 12:16:19 CEST 2003

[user[adel15 user] $\$$ cd attack

ini-kernel-backdoor

linux-brk, c linux-ptrace-2,c

[user[adel15 attacks] : make linux-ptrace-1

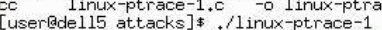

$[+]$ Start

+] Attached to 2314

+ + Shell caught

[+] Now wait for suid shell...

[+] Start

goubaultadel15\{reports 1010 : ssh user@iocalhost

userdlocalhost's password:
Last login: Fri Oct 15 10:43:04 2004 from localhost. localdomain
Kickstart-installed Red Hat Linux Fri Sep 26 12:16:19 CEST 2003

Kickstart-installed Red Hat Linux Fri Sep 26 12:16:19 CEST 2003

goubaultadel15\{reports\} 1011:

20.

Fig. 3. ORCHIDS counters the ptrace attack 
When ORCHIDS is enabled, the same attack produces the response shown in Figure 3. The illegal connection is closed, and the account is closed (the intruder cannot $\log$ in again). The reason for the latter is that the attack actually succeeded, and there is a risk that the intruder managed to install a setuid bit shell, which would allow him to regain root privileges without even replaying an attack.

Figure 4 shows excerpts from the detailed report that ORCHIDS produces. Look at the first event that matched the attack (top). This is the call to ptrace (see field 23, rawsnare.syscall) using the ATTACH command (see field 50, rawsnare.ptrace_req) by process 2987 (field 28, rawsnare. pid) with effective uid 501 (field 24, rawsnare. euid) on process 2988 (field 51, rawsnare.ptrace_pid). The last event matching the attack, namely, the DETACH event is event 6 in the report (not shown).

Compared to the idealized formula (1), the formula contains additional transitions to track actions that follow the last characteristic event of the attack. Event 7 (not shown) in particular is a call from the (malicious) shellcode to chown, and event 8 (shown) is a call from the shellcode to chmod, indicating that the intruder managed to install the setuid bit on some (now root-owned) file before it got kicked out by ORCHIDS: the value of the protection bits (field 35, rawsnare. createmode) is decimal 3565, i.e. octal 6755 , aka. $-r w s r-s r-x$ in more readable Unix notation. This confirms our guess (above) that the intruder produced a setuid bit shell.

\section{A.2 Status}

ORCHIDS is currently in a prototype status. It is deployed experimentally in the network of the laboratory where it was designed and implemented (LSV), consisting of about 150 equipments (servers, workstations and routers). It supports common standards to make deployment easier (Unix syslog, sendmail, apache logs, snmp protocol, etc.) ORCHIDS is designed so as to run as autonomously as possible. Naturally, it still requires a systems or security administrator.

It has been deployed for local, real-conditions testing on the network of the lab since September, 2004. We do not want to publicize this yet, as this kind of announcement invariably attracts script kiddies. Since ORCHIDS is still a prototype, this would only augment the chances that our network be hacked into.

\section{A.3 Availability}

As of today, ORCHIDS is not available in source form. The single-laptop demonstration version is however available free of charge for academic use only, in binary form (compiled for Linux with glibc; current version is known to work on Linux 2.4.18-3, 2.4.20-20.7, and 2.4.20-24.7), upon request to the authors.

The attacks demonstrated are known to work on Linux 2.4.18-3, Red Hat release 7.3 (Valhalla). The ptrace attack will not work on higher versions. In general, the demonstrated versions will not work on up-to-date versions of Linux: we will not demonstrate attacks that have not been countered already.

The mod_ssl attack (a network attack allowing an intruder to gain access to a remote computer, which we shall demonstrate as a preparation for launching a ptrace 
- orchids -

Report for rule: ptrace

\begin{tabular}{|c|c|c|c|c|}
\hline \multicolumn{5}{|c|}{ Event 1 (id:0x80e7820 1 ref) } \\
\hline FID & Field & Type & Monotony & Data content \\
\hline 53 & rawsnare.ptrace_data & ptr32 & unkn & (nil) \\
\hline 52 & rawsnare.ptrace_addr & ptr32 & unkn & (nii) \\
\hline 51 & rawsnare.ptrace_pid & int & unkn & 2988 \\
\hline 50 & rawsnare.ptrace_req & vstr & unkn & (16) PTRACE_ATTACH \\
\hline 31 & rawsnare retcode & int & unkn & 0 \\
\hline 30 & rawsnare.procname & vstr & unkn & linux-ptrace-1 \\
\hline 29 & rawsnare.ppid & int & unkn & 2986 \\
\hline 28 & rawsnare.pid & int & unkn & 2987 \\
\hline 27 & rawsnare.egid & int & unkn & 501 \\
\hline 26 & rawsnare.euid & int & unkn & 501 \\
\hline 25 & rawsnare.rgid & int & unkn & 501 \\
\hline 24 & rawsnare.ruid & int & unkn & 501 \\
\hline 23 & rawsnare.syscall & vstr & unkn & (26) SYS_ptrace \\
\hline 22 & rawsnare.class & int & unkn & 10 \\
\hline 21 & rawsnare time & timeval & mono & $\begin{array}{l}\text { Fri Oct } 1510: 50: 502004+235177 \text { us } \\
(1097830250.235177)\end{array}$ \\
\hline 13 & udp.msg & bstr & unkn & 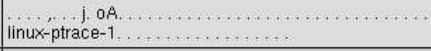 \\
\hline 12 & udp.dst_port & int & unkn & 6262 \\
\hline 9 & udp.src_addr & ipv4 & unkn & 127.0.0.1 (localhost.localdomain, localhost) \\
\hline 8 & udp time & timeval & mono & $\begin{array}{l}\text { Fri Oct } 1510: 50: 502004+235190 \text { us } \\
(1097830250.235190)\end{array}$ \\
\hline 7 & udp.event & int & mono & 566 \\
\hline
\end{tabular}

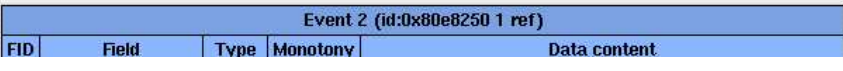

$\bullet$

\begin{tabular}{|c|c|c|c|c|}
\hline 3 & uup.sre_auur & jpv4 & Jurikn & 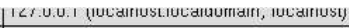 \\
\hline 8 & udp.time & timeval & mono & $\begin{array}{l}\text { Fri Oct } 1510: 50: 502004+370506 \text { us } \\
(1097830250.370506)\end{array}$ \\
\hline 7 & udp.event & int & mono & 590 \\
\hline
\end{tabular}

\begin{tabular}{|c|c|c|c|c|}
\hline \multicolumn{5}{|c|}{ Event 8 (id:0x80e89a0 2 ref) } \\
\hline FID & Field & Type & Monotony & Data content \\
\hline 35 & rawsnare.createmode & int & unkn & 3565 \\
\hline 34 & rawsnare mode & int & unkn & 0 \\
\hline 33 & rawsnare.path & vstr & unkn & /home/user/attacks/linux-ptrace-1 \\
\hline 32 & rawsnare.workdir & vstr & unkn & 1 \\
\hline 31 & rawsnare retcode & int & unkn & 0 \\
\hline 30 & rawsnare procname & vstr & unkn & modprobe \\
\hline 29 & rawsnare.ppid & int & unkn & 2986 \\
\hline 28 & rawsnare pid & int & unkn & 2988 \\
\hline 27 & rawsnare.egid & int & unkn & 0 \\
\hline 26 & rawsnare euid & int & unkn & 0 \\
\hline 25 & rawsnare.rgid & int & unkn & 501 \\
\hline 24 & rawsnare ruid & int & unkn & 0 \\
\hline 23 & rawsnare.syscall & vstr & unkn & (15) SYS_chmod \\
\hline 22 & rawsnare.class & int & unkn & 1 \\
\hline 21 & rawsnare time & timeval & mono & $\begin{array}{l}\text { Fri Oct } 1510: 50: 502004+298358 \text { us } \\
(1097830250.298358)\end{array}$ \\
\hline 13 & udp.msg & bstr & unkn & modprobe . . . . . . . home/user/attacks/linux-ptrace-1 d \\
\hline
\end{tabular}

Fig. 4. Report on the ptrace attack 
attack; not described here for space reasons) requires the Apache Web server v.1.3.2311 (exactly), the mod_ssl-2.8.7-4 Apache SSL extension (exact version again), and the cryptographic package openss $1-0.9 .6 \mathrm{~b}-18$ (again, exact version).

We do not wish to distribute the attacks, whether in source or binary code, for obvious reasons.

See the Web page http: //www. Isv.ens-cachan.fr/orchids/for more information on ORCHIDS. 